\section{Utilisation de la souche B19 dans la prophylaxie médicale de la brucellose bovine au Nord-Cameroun. Etude de l'effet de la dose sur le taux et la durée de séroconversion chez des femelles zébus}
A. Martrenchar ${ }^{1^{*}}$
D. Bouchel ${ }^{2 * *}$
B.M. Njanpop'

A. Yaya ${ }^{1}$

MARTRENCHAR (A.), BOUCHEL (D.), NJANPOP (B.M.), YAYA

(A.).. Utilisation de la souche B19 dans la prophylaxie médicale de la brucellose bovine au Nord-Cameroun. Etude de l'effet de la dose sur le taux et la durée de séroconversion chez des femelles zébus. Revue Élev. Méd. vét. Pays trop., 1995, 48 (1) : 37-40

Quatre troupeaux de zébus du Nord-Cameroun, comprenant en tout 136 animaux, ont été vaccinés par voie sous-cntanée avec les doses suivantes de la souche $\mathrm{B} 19: 5 \times 10^{9}$ unités formant colonies (UFC), $10^{9} \mathrm{UFC}, 5 \times 10^{8}$ UFC et $10^{7}$ UFC. Vingt-huit jours après la vaccination, les taux de séroconversion ont été respectivement les suivants : 97,4, 96,2, 84,2 et 73,3 p. 100. Sur les 52 animaux, dont 39 âgés de plus d'un an le jour de la vaccination, ayant pu faire l'objet d'un contrôle sérologique ultérieur, 1 seul présentait des anticorps 6 mois après la vaccination. A la dose de $10^{\circ}$ UFC, le coût de revient du vaccin B19 produit au Laboratoire national vétérinaire de Boklé est de $65 \mathrm{~F}$ CFA la dose ; à la dose habituellement préconisée de $5 \times 10^{10}$ UFC, il est de $1740 \mathrm{~F}$ CFA. Les différentes perspectives d'utilisation de la souche B19 dans la prophylaxie médicale contre la brucellose bovine au Nord-Cameroun sont discutées.

Mots clés : Bovin - Zébu - Brucellose - Brucella abortus - Contrôle de maladie - Vaccin - Coût - Cameroun.

\section{Introduction}

La brucellose bovine est une des maladies infectieuses les plus importantes du cheptel bovin du Nord-Cameroun. Les estimations des taux de prévalences individuelles varient selon les études de 12 à 25 p. 100 (11, 16). Dans une région où les animaux ne sont pas identifiés et où l'Etat n'est pas en mesure d'indemniser les éleveurs pour l'élimination des animaux contaminés, seule une prophylaxie médicale est envisageable. Parmi les différents vaccins disponibles, la souche de Brucella

1. Laboratoire national vétérinaire de Boklé, BP 503 Garoua, Cameroun.

2. Institut de Recherches zootechniques et vétérinaires, Station de Garoua, BP 1073, Garoua, Cameroun.

* Adresse actuelle : 24 rue Calvé, 33000 Bordeaux, France (du 1.07.95 au 1.09.95). A compter du 1.09.95: Laboratoire central de recherches avicole et porcine, BP 53, Zoopole des Côtes d'Armor, 22440 Ploufragan, France.

** Adresse actuelle: CIRAD-EMVT, 10 rue Pierre Curie, 94704 Maisons-Alfort Cedex, France.

Reçu le 27.5.1994, accepté le 21.3.1995. abortus B19 présente l'avantage de ne nécessiter qu'une seule injection sous-cutanée en primo-vaccination. Les normes internationales (19) recommandent l'utilisation de ce vaccin à un titre très élevé $\left(5 \times 10^{10}\right.$ à $8 \times 10^{10}$ unités formant colonie (UFC)). A ce titre, le coût de la dose unitaire (1 600 F CFA, vaccin ABORSEC Rhône-Mérieux, prix hors taxe départ France) est financièrement inacceptable pour l'éleveur du Nord-Cameroun. Dans les années 80, de nombreuses études se sont intéressées à l'utilisation de la souche $\mathrm{B} 19$ à des doses réduites $(2,3,4,5,6,8,9$, $10,12,13,14,17,18,20)$. L'efficacité de ce vaccin est grandement dépendante des conditions locales et des races d'animaux (14). Cette étude est menée afin d'effectuer une première approche sur la possibilité d'utiliser des doses réduites du vaccin B19 par voie sous-cutanée sur le cheptel bovin du Nord-Cameroun.

\section{Matériel et Méthodes}

\section{Production du vaccin}

La souche B19 a été cuitivée au Laboratoire national vétérinaire de Boklé sur gélose pomme de terre selon un protocole décrit par ailleurs (1). Le support de lyophilisation utilisé était le lait stérilisé UHT. Le titre après lyophilisation était de $2.10^{10} \mathrm{UFC} / \mathrm{ml}$. La reconstitution et les dilutions du vaccin se faisaient avec de l'eau physiologique stérile sur glace pilée en 2 ou 3 manipulations successives juste avant l'injection.

\section{Choix des animaux}

Les animaux étaient des zébus de races locales Akou (White Fulani), M'bororo (Red Fulani), Goudali et Arabe Choa. Ils étaient élevés selon le mode traditionnel sans contrôle sur la reproduction. Les mâles étaient commercialisés vers l'âge de 3 ans et l'âge moyen à la première mise bas était de 45 mois. Les animaux identifiés par une boucle auriculaire étaient répartis en 4 troupeaux. Trois de ces troupeaux avaient fait l'objet depuis trois an's d'une prophylaxie sanitaire contre la brucellose (contrôle sérologique du cheptel et des entrées suivi de l'élimination des animaux positifs). Tous les animaux étaient des femelles, non gestantes d'après l'avis de l'éleveur.

\section{Séances de vaccination et suivis sérologiques}

Huit séances de vaccination réparties sur une année ont été effectuées. Les animaux étaient contrôlés sérologiquement le jour de la vaccination et seuls les animaux négatifs à ce premier contrôle (136 animaux) furent inclus dans l'analyse. Les animaux étaient contrôlés une nouvelle fois, 4 semaines après la vaccination. Cinquante-deux animaux purent être contrôlés de nouveau entre 
3 et 6 mois après la vaccination. Les séances de vaccination avec les doses vaccinales utilisées, les âges et les races des animaux, ainsi que les animaux recontrôlés entre trois et six mois après la vaccination sont indiqués dans le tableau I.

\section{Tests sérologiques}

Tous les animaux furent testés à l'aide de l'épreuve à l'antigène tamponné (antigène Bengatest, Rhône Mérieux, Lyon, France) et du test de fixation du complément en tubes (antigène Antifix, Rhône Mérieux, Lyon, France). Pour ce dernier test, le seuil de positivité choisi fut de 1/8 (40 Ul/ml). Les sérums étaient considérés positifs s'ils réagissaient à l'un ou l'autre des deux tests.

\section{Calcul du prix de revient du vaccin}

Aux coûts des matières premières et du conditionnement, ont été rajoutés les frais généraux du Laboratoire national vétérinaire de Boklé-Garoua. Ceux-ci incluaient les salaires du personnel, les frais fixes de fonctionnement (électricité, entretien des locaux et des équipements), ainsi que l'amortissement des locaux et des équipements. Ceci a été fait sur la base du calcul des coûts de revient des autres vaccins produits au Laboratoire national vétérinaire de Boklé* $(7)$. Pour des vaccins titrant $10^{9}$ UFC, la production annuelle a été déterminée à partir du marché potentiel alors que, pour un titre de $5 \times 10^{10}$, elle était limitée par les capacités locales compte tenu des autres productions.

\section{Analyse des données}

La variable illustrative était l'existence d'une séroconversion à $28 \mathrm{j}$ ( 2 modalités). Plusieurs variables explicatives dichotomiques ont été créées : dose vaccinale supérieure ou égale à $5 \times 10^{9}, 10^{\circ}$ et $5 \times 10^{8}$ UFC ( 3 variables), race des animaux (4 variables) et la classe d'âge (1 variable à 2 modalités : supérieur ou inférieur à un an). L'analyse des tableaux croisés $2 \times 2$ s'est faite à l'aide du test de Fisher exact.

\section{Résultats}

\section{Séroconversion 28 jours après la vaccination}

Les résultats sont présentés dans le tableau I. L'analyse statistique a montré que seules les variables relatives à la dose vaccinale étaient significatives. Le plus haut degré de signification a été obtenu avec la variable "dose

\footnotetext{
* Le détail du calcul des coûts de production peut être demandé au Dr A. Martrenchar, Laboratoire central de recherches avicole et porcine, BP 53, Zoopole des Côtes d'Armor, 22440 Ploufragan, France.
}

vaccinale supérieure ou égale à $10^{9} \mathrm{UFC} / \mathrm{ml} "(p<0,003)$. Les résultats en fonction de la dose vaccinale sont présentés dans le tableau II.

\section{Persistance des anticorps dans les 3 à 6 mois après la vaccination}

Sur les 52 animaux ayant pu être suivis, un seul présentait des anticorps anti-Brucella 6 mois après la vaccination.

\section{Calcul du prix de revient du vaccin}

En produisant des flacons de 100 doses titrant $10^{9}$ UFC par dose, le coût de revient est de 65 F CFA la dose, sur la base d'une production annuelle de 400000 doses (128 F CFA sur la base d'une production annuelle de 100000 doses). En produisant des flacons de deux doses titrant $5 \times 10^{10}$ UFC par dose, le coût de revient est de $1740 \mathrm{~F}$ CFA la dose (sur la base d'une production annuelle de 24000 doses).

\section{Discussion}

Dans un des troupeaux de l'étude, sur 12 animaux prélevés, 3 étaient positifs le jour de la vaccination (troupeau d'animaux de race Goudali). Ce troupeau faisait l'objet d'un contrôle sérologique depuis trois ans et aucune explication n'a pu être trouvée avec certitude. Les trois animaux en question ont été éliminés.

Le seuil de séropositivité retenu en fixation de complément a été de $40 \mathrm{Ul} / \mathrm{ml}$. Le seuil habituellement retenu en Europe est de $20 \mathrm{UI} / \mathrm{ml}$. Trois animaux vaccinés à la dose de $10^{7} \mathrm{UFC}$ étaient positifs à $\mathrm{J} 28$ à ce seuil mais négatifs au seuil de $40 \mathrm{Ul} / \mathrm{ml}$ ainsi qu'à l'épreuve à l'antigène tamponné. Si l'on prend le seuil de $20 \mathrm{Ul} / \mathrm{ml}$ comme base de l'analyse, la variable "dose vaccinale supérieure ou égale à $10^{\circ}$ UFC" reste significative $(p<0,004)$.

Le pourcentage d'animaux présentant une séroconversion augmente avec la dose vaccinale. Ceci est conforme aux résultats d'une autre étude (9) mais néanmoins insuffisant pour en tirer des conclusions sur l'état de protection des animaux. En effet, il a été montré que la protection contre la brucellose était due à une immunité de type cellulaire. II a par ailleurs été suggéré que la séroconversion et l'immunité de type cellulaire pourraient être toutes les deux liées à la dose vaccinale mais selon des médiateurs différents (9). Dans le cas de la brucellose, il n'existe pas de technique fiable permettant de mesurer l'immunité de type cellulaire $(8,9)$. La seule méthode acceptée pour doser l'immunité globale consiste à inoculer par voie conjonctivale des animaux vaccinés avec une souche virulente et à tenter d'isoler la souche d'épreuve à la mise bas ou à l'autopsie. Les résultats sont grandement dépendants de la virulence de la souche et de la dose d'épreuve (8). 
TABleau I

Résultats des mesures de la séroconversion sur 4 troupeaux de zébus du Nord-Cameroun vaccinés avec différentes doses du vaccin B19 en fonction de la race et de la classe d'âge
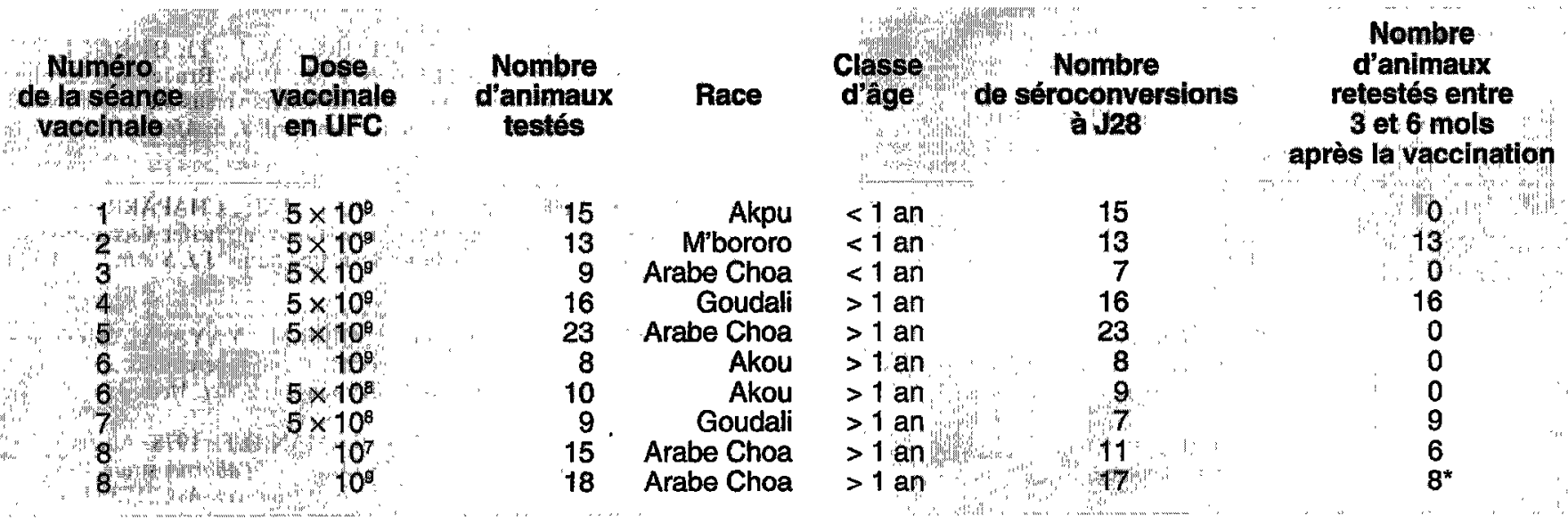

Un animal était positif à l'épreuve à l'antigène tamponné 6 mois après la vaccination.

TABLEaU II

Pourcentages de séroconversion 28 jours après la vaccination en fonction des doses du vaccin B19

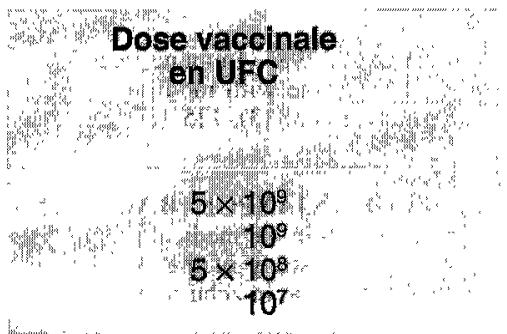

$\begin{gathered}\text { Nombre } \\ \text { d'animaux } \\ \text { testés }\end{gathered}$

76
26
19
15

Les résultats du suivi sérologique indiquent que jusqu'à une dose de $5 \times 10^{9}$ UFC, la vaccination, même celle des adultes, ne perturbe pas notablement le diagnostic sérologique au-delà d'un délai de 6 mois. Ce résultat devrait néanmoins être confirmé sur un plus grand nombre d'animaux. Les études sur le problème de la persistance d'anticorps suite à la vaccination par des doses faibles du vaccin B19 donnent des résultats variables selon les auteurs $(2,6,9,12,14,15,18)$. II est généralement admis que ce problème est plus important chez les animaux subissant un rappel de vaccination et chez les femelles gestantes. Pour pallier cet inconvénient, il est possible soit de vacciner uniquement les jeunes animaux, soit d'utiliser des doses réduites par voie conjonctivale. La vaccination des jeunes peut être efficace dans des régions à faible taux d'infection. Au Nord-Cameroun, la présence régulière de foyers épizootiques devrait entraîner la vaccination de toutes les femelles alentour. La vaccination par voie conjonctivale a l'inconvénient de demander une contention plus rigoureuse par rapport à la voie sous-cutanée et nécessite deux interventions en primo-vaccination. L'utilisation de doses réduites en souscutané pourrait être une solution alternative intéressante.
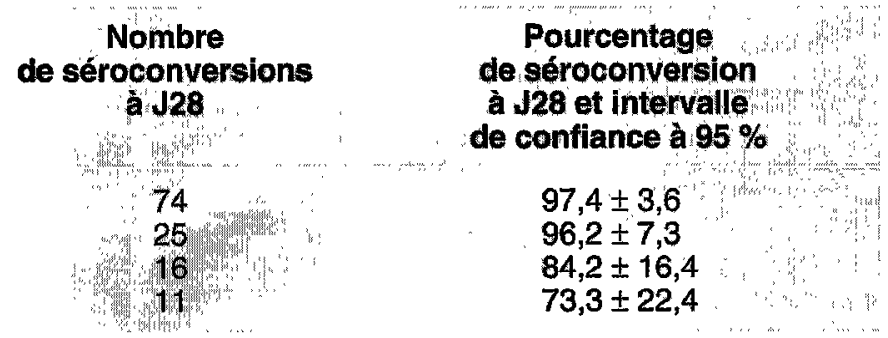

Le coût de revient de la dose vaccinale produite sur place rend prohibitive l'utilisation du vaccin à la dose habituellement conseillée $\left(5 \times 10^{10}\right.$ à $8 \times 10^{10}$ UFC). En revanche, à la dose de $10^{9}$ UFC, le laboratoire producteur pourrait vendre ce vaccin au prix de 65 F CFA la dose. Ceci est supportable financièrement par l'éleveur. A titre de comparaison, le prix actuel du vaccin contre le charbon bactéridien à la sortie du Laboratoire national vétérinaire de Boklé est de 28 F CFA la dose.

\section{Conclusion}

A la dose de $10^{\circ}$ UFC, la souche B19 provoque une importante séroconversion sur les zébus de l'expérimentation. A cette dose, le laboratoire peut proposer le vaccin à un prix acceptable pour l'éleveur. Afin de préciser le niveau de protection conféré par la souche B19 et de proposer une stratégie vaccinale contre la brucellose bovine, d'autres études, incluant des épreuves virulentes et des mesures de l'influence de vaccinations en milieu traditionnel sur les paramètres zootechniques du troupeau, sont nécessaires. 


\section{Bibliographie}

1. ALTON G.G., JONES L.M., PIETZ D.E., 1977. La brucellose. Techniques de laboratoire (2 éd). Genève, Suisse, Organisation Mondiale de la Santé, p. 145-153.

2. ALTON G.G., CORNER L.A., PLACKETT P., 1980. Vaccination of pregnant cows with low doses of Brucella abortus strain 19 vaccine. Aust. vet. J., 56: 369-372

3. ALTON G.G., CORNER L.A., 1981. Vaccination of heifers with a reduced dose of Brucella abortus strain 19 vaccine before first mating. Aust. vet. J., 57: 548-550

4. BARTON C.E., LOMME J.R., 1980. Reduced-dose whole herd vaccination against brucellosis: a review of recent experience. J. Am. vet. Ass., 12: $1218-1220$

5. BECKETT F.W., MacDIARMID S.C., 1985. The effect of reduced dose Brucella abortus strain 19 vaccination in accredited dairy herds. Br. vet. $J$., 1985, 141: 507-514.

6. BECKETT F.W., MacDIARMID S.C., 1987. Persistent serological titres following reduced dose Brucella abortus strain 19 vaccination. Br. vet. J., 143: 477-479.

7. BENITES A., GUILLOTEAU B., MARTIN A., 1993. Mission d'expertise pour la définition de la politique d'intervention du Laboratoire National Vétérinaire de Boklé-Garoua. Maisons-Alfort, France, CIRAD-EMVT, $142 \mathrm{p}$.

8. CONFER A.W., HALL S.M., FAULKNER C.B., ESPE B.H., DEYOE B.L., MORTON R.J., SMITH R.A., 1985. Effects of challenge dose on the clinical and immune responses of cattle vaccinated with reduced doses of Brucella abortus strain 19. Vet. Microbiol., 10: 561-575.

9. CRAWFORD R.P., ADAMS L.G., RICHARDSON B.E., 1990. Effect of dose of Brucella abortus strain 19 in yearling heifers on the relative risk of developing brucellosis from challenge exposure with strain 2308. Am. J. vet. Res., 51 (11): 1837-1840.

10. DAVIES G., COCKS E., HEBERT N., 1980. Brucella abortus (Strain 19) vaccine: (a) Determination of the minimum protective dose in cattle; (b) The effect of vaccinating calves previously inoculated with anti-Brucella abortus serum. J. Biol. Stand., 8: 165-175.

11. DOMENECH J., LUCET Ph., VALLAT B., STEW $A R T$ Ch., BONNET J.B., HENTIC A., 1982. La brucellose bovine en Afrique centrale. III. Résultats statistiques des enquêtes menées au Tchad et au Cameroun. Revue Élev. Méd. vét. Pays trop., 35 (1) : 15-22.

12. ERASMUS J.A., ERASMUS M.C., 1987. The use of reduced-dose Brucella abortus strain 19 in the control of bovine brucellosis. J. S. Afr. vet. Ass., 58 (2): 71-75.
13. FLORES-CASTRO R, FERNANDEZ De CORDOBA L., TREJOSALOMON J., Del RIO-VARGAS J., 1985. Adult cattle vaccination and revaccination with strain 19 reduced dosis for the control of brucellosis: a field-experience in Mexico. Int. I. Zoon., 12: 299-303.

14. HERR S., BOSMAN P.P., EHRET W.J., TE BRUGGE L.A., WILLIAMSON C.C., PIETERSON P.M., 1986. Brucellosis serology: reduced dose S19 vaccination of yearling heifers versus the use of the standard dose at 5-7 months of age in a clean herd. J. S. Afr. vet. Ass.; 57 (4): 215-219.

15. HERR S., EHRET W.J., RIBEIRO L.M.M. , CHAPARRO F., 1990. The persistence of serological reactions following heifer vaccination with Brucella abortus strain 19 on brucellosis free farms. J J. S. Afr. vet. Ass., 61 (1): 11-13.

16. MARTRENCHAR A., NJANPOP B.M., YAYA A., NJOYA A., TULASNE J.J., 1993. Problems associated with tuberculosis and brucellosis skin-test methods in northern Cameroon. Prev. Vet. Med., 15 : 221-229.

17. NICOLETTI P., JONES L.M., BERMAN D.T., 1978. Adult vaccination with standard and reduced doses of Brucella abortus strain 19 vaccine in a dairy herd infected with brucellosis. J. Am. vet. Ass., 173 (11): 14451449.

18. ODEON A.C., CAMPERO C.M., MOREIRA A.R., SALAMANCO A.G., MADRID C.R., ZAMORA A.S., 1987. Revaccination with a reduced dose of Brucella abortus strain 19 vaccine of breeding cows in the Pampas region of Argenlinla. Revue sci. tech. Off. int. Epizoot., 6 (4): 1063-1071.

19. Office International des Epizooties, 1992. Manual of standards for diagnostic tests and vaccines. Paris, France, p. 260-276.

20. VENKATESHA M.D., UPADHYE A.S., 1987. Immune response of young calves to Brucella abortus S. 19 vaccine with modified method. Indian J. Comp. Microbiol. Immunol. Infect. Dis., 8 (3): 125-129.

MARTRENCHAR (A.), BOUCHEL (D.), NJANPOP (B.M.), YAYA (A.). The use of the Brucella abortus strain 19 in the medical prophylaxis of bovine brucellosis in northern Cameroon: study of the effect of the vaccinal dose on seroconversion rate and duration in zebu females. Revue Élev. Méd. vét. Pays trop., 1995, 48 (1): 37-40

Four herds of zebus from northern Cameroon totalling 136 animals were vaccinated subcutaneously with the following doses of Brucella abortus strain 19: $5 \times 10^{9}$ colony-forming units (CFU), $10^{9} \mathrm{CFU}, 5 \times 10^{8} \mathrm{CFU}$ and $10^{7} \mathrm{CFU}$. Twenty-eight days after vaccination, the following seroconversion rates were observed respectively: $97.4,96.284 .2$ and $73.3 \%$. Of the 52 animals which could be tested subsequently including 39 over one year old on the vaccination day, only one showed antibodies 6 months after vaccination. The cost price of the strain 19 vaccine produced at the Boklé National Veterinary Laboratory was estimated to be 65 F CFA at the $10^{\circ} \mathrm{CFU}$ dose and $1,740 \mathrm{~F}$ CFA at the $5 \times 10^{10} \mathrm{CFU}$ dose usually recommended. The use of the Buck 19 strain in the medical prophylaxis of bovine brucellosis in northern Cameroon is discussed.

Key words: Cattle - Zebu cattle - Brucellosis - Brucella abortus - Disease control - Vaccine - Cost - Cameroon. 